\title{
iccafd
}

Revista Iberoamericana de Ciencias de la Actividad Fisica y el Deporte

\section{EL MASAJE EN LA PREVENCIÓN Y TRATAMIENTO DEL DOLOR MUSCULAR TARDÍO: UNA REVISIÓN SISTEMÁTICA ACTUALIZADA}

\section{MASSAGE IN THE PREVENTION AND TREATMENT OF DELAYED ONSET MUSCLE SORENESS: AN UPDATED SYSTEMATIC REVIEW}

Candia Luján, R. ${ }^{1}$, Paredes Carrera, RA. ${ }^{2}$, Costa Moreira, $0 .{ }^{3}$, Candia Sosa, KF. ${ }^{4}$, De Paz

Fernández, JA. ${ }^{5}$

${ }^{1}$ Candia Luján, R. Doctor en Ciencias de la Actividad Física y el Deporte, Universidad Autónoma de Chihuahua, México.rcandia@uach.mx

${ }^{2}$ Paredes Carrera, RA. Maestro en Entrenamiento Deportivo, Universidad Autónoma de Chihuahua, México.rparedes@uach.mx

${ }^{3}$ Costa Moreira, O. Doctorado en Ciencias de la Actividad Física, Universidad Federal de Viçosa, Brasil. osvaldo-moreira@ufv.br

${ }^{4}$ Candia Sosa, KF. Licenciatura en Educación Física, Universidad Autónoma de Chihuahua, México.kev_fer_17@outlook.com

${ }^{5}$ De Paz Fernández, JA. Doctor en Medicina y Cirugía, Universidad de León, España. japazf@unileon.es

Código UNESCO: 2411

Clasificación Consejo de Europa: Fisiología del Ejercicio

Correspondencia: Candia Luján, R. rcandia@uach.mx

\section{RESUMEN}

El masaje es una de las terapias más utilizadas para aliviar el dolor muscular tardío (DMT). El objetivo del presente estudio fue determinar la efectividad del masaje en el tratamiento del DMT, para lo cual se llevó a cabo una revisión sistemática en las bases de datos, Pubmed, Scopus, SportDiscus, Web of Science y el buscador Google académico, usando las palabras clave delayed onset muscle soreness y exercise induced muscle damage combinado con massage. Se incluyeron en el estudio 23 artículos en los cuales el 78\% 
mostró disminución del DMT mientras que en el restante $22 \%$ no hubo mejoras o bien empeoró. El análisis de los estudios permite concluir que el masaje es una terapia efectiva en el tratamiento del dolor muscular tardío.

Palabras clave: masaje, dolor muscular tardío, terapia, revisión sistemática.

\section{ABSTRACT}

Massage is one of the most widely used therapies for relieving delayed onset muscle soreness (DOMS). The aim of the present study was to determine the effectiveness of massage in the treatment of DOMS, for which a systematic review was carried out in the databases, Pubmed, Scopus, SportDiscus, Web of Science and the Google Scholar search engine using the words key words delayed onset muscle soreness and exercise induced muscle damage combined with massage. This study included 23 articles in which $78 \%$ showed a decrease in DOMS while in the remaining $22 \%$ there were no improvements or gotten worse. The analysis of these studies allows to conclude that massage is an effective therapy in the treatment of delayed onset muscle soreness.

Key words: massage, delayed onset muscle soreness, therapy, systematic review

\section{INTRODUCCIÓN}

El dolor muscular tardío (DMT) se clasifica como una distensión muscular tipo I1. Este dolor es el que se presenta entre ocho y doce horas después de haber realizado una actividad física desacostumbrada, siendo entre las 24 y 72 horas cuando se presenta su mayor intensidad, dicho dolor puede durar entre 5 y 7 días²$^{2}$. El dolor es mayor cuando en la actividad física están involucradas más acciones musculares excéntricas, como bajar por una escalera, bajar un peso, entre otras ${ }^{3}$. Aunque es un fenómeno muy común, ya que la gran mayoría de las 
personas lo ha sufrido en algún momento, aún no se conoce su etiopatogenia. Sin embargo, a través de los años han sido propuestas diversas teorías que han tratado de darle respuesta 4 . Entre las teorías se encuentran la del ácido láctico, del espasmo muscular, del daño del tejido conectivo, del daño muscular, de la inflamación y de la extravasación de enzimas. Las dos primeras han sido puestas en duda y desechadas por diversos investigadores en sus estudios ${ }^{5}$. Para Cheung et al. ${ }^{5}$ la explicación del DMT no puede ser dada por una sola teoría, ya que mencionan que es una secuencia de eventos que integra aspectos de diversas teorías. Por su parte Connolly et al. ${ }^{6}$ describen el mecanismo de la producción del DMT de la siguiente manera: el ejercicio excéntrico daña la membrana celular de la fibra, lo que activa una respuesta inflamatoria, que a su vez conduce a la síntesis de prostaglandinas (PGE2) y leucotrienos. Las PGE2 causan la sensación de dolor por la sensibilización de las fibras aferentes tipo III y IV a los estímulos químicos, mientras los leucotrienos incrementan la permeabilidad vascular, lo que permite que los neutrófilos lleguen al sitio dañado. El "estallido respiratorio" de los neutrófilos genera radicales libres, los cuales pueden agravar el daño de la membrana celular. La inflamación resulta del movimiento de células y fluidos del torrente sanguíneo al espacio intersticial, lo que puede contribuir a la sensación de dolor.

Los mecanismos propuestos han permitido a numerosos investigadores estudiar diversas estrategias para prevenir y/o tratar los síntomas relacionados con el DMT, restablecer tan rápido como sea posible la fuerza muscular y/o reducir la lesión inicial ${ }^{7}$. Las intervenciones propuestas pueden ser agrupadas en tres categorías: ${ }^{6}$ a) tratamientos farmacológicos usando antiinflamatorios no esteroideos $^{8}$, b) el uso de suplementos alimenticios ${ }^{9}$, y c) tratamientos de terapia física ${ }^{10}$. En esta última categoría sobresale, por la gran cantidad de estudios realizados, el uso del masaje. Por lo que surge la siguiente pregunta ¿es efectivo el masaje en la prevención y el tratamiento del DMT?

Por lo que se propuso como objetivo llevar a cabo una revisión de estudios que se han realizado en los últimos años, donde se haya usado el masaje como estrategia para prevenir o tratar el DMT. Para garantizar la calidad de los 
estudios analizados en este trabajo la búsqueda se realizó en las principales bases de datos electrónicas relacionadas con la biomedicina y el deporte.

\section{MÉTODO}

Para la elaboración de la presente revisión se tomó como guía el artículo de Guirao-Goris et al. ${ }^{11}$. Para la identificación de los estudios incluidos en esta revisión se llevó a cabo una búsqueda en las siguientes bases de datos: Pubmed, Scopus, SportDiscus y la Web of Science (WOS), además del buscador Google Académico. La búsqueda se realizó entre enero y febrero de 2017. Las palabras clave utilizadas fueron; delayed onset muscle soreness, exercise induced muscle damage, los acrónimos DOMS y EIMD combinados con massage. Los criterios de inclusión: que los estudios fueran hechos en humanos, publicados entre los años 2000 al 2017, en revistas de revisión por pares, que la aplicación del masaje no se combinara con otra terapia, aunque sí que fueran comparadas, y por último, que el masaje fuera aplicado con las manos o con algún dispositivo.

Para la selección de los artículos, en primer lugar se analizaba el título, si en éste aparecía la relación entre el masaje y el DMT, entonces se revisaba el resumen y se recuperaba el artículo en extenso para hacer el análisis respectivo.

\section{RESULTADOS}

En la figura 1 se detalla el procedimiento de la selección de los estudios. En total se incluyeron 23 artículos que cumplieron con los criterios de inclusión establecidos para este estudio. De los estudios incluidos, en $78 \%$ se presentó una disminución del DMT con el masaje, en el $18 \%$ no hubo efectos y en el restante $4 \%$ el dolor se agravó. El tiempo utilizado en la aplicación del masaje fue diverso, desde cinco minutos hasta la media hora, el promedio fue de $16 \mathrm{~min}$. En el $65 \%$ de los estudios el masaje estuvo aplicado por personas capacitadas. En aproximadamente el $70 \%$ de los estudios el diseño de investigación fue aleatorio usando grupo control. 


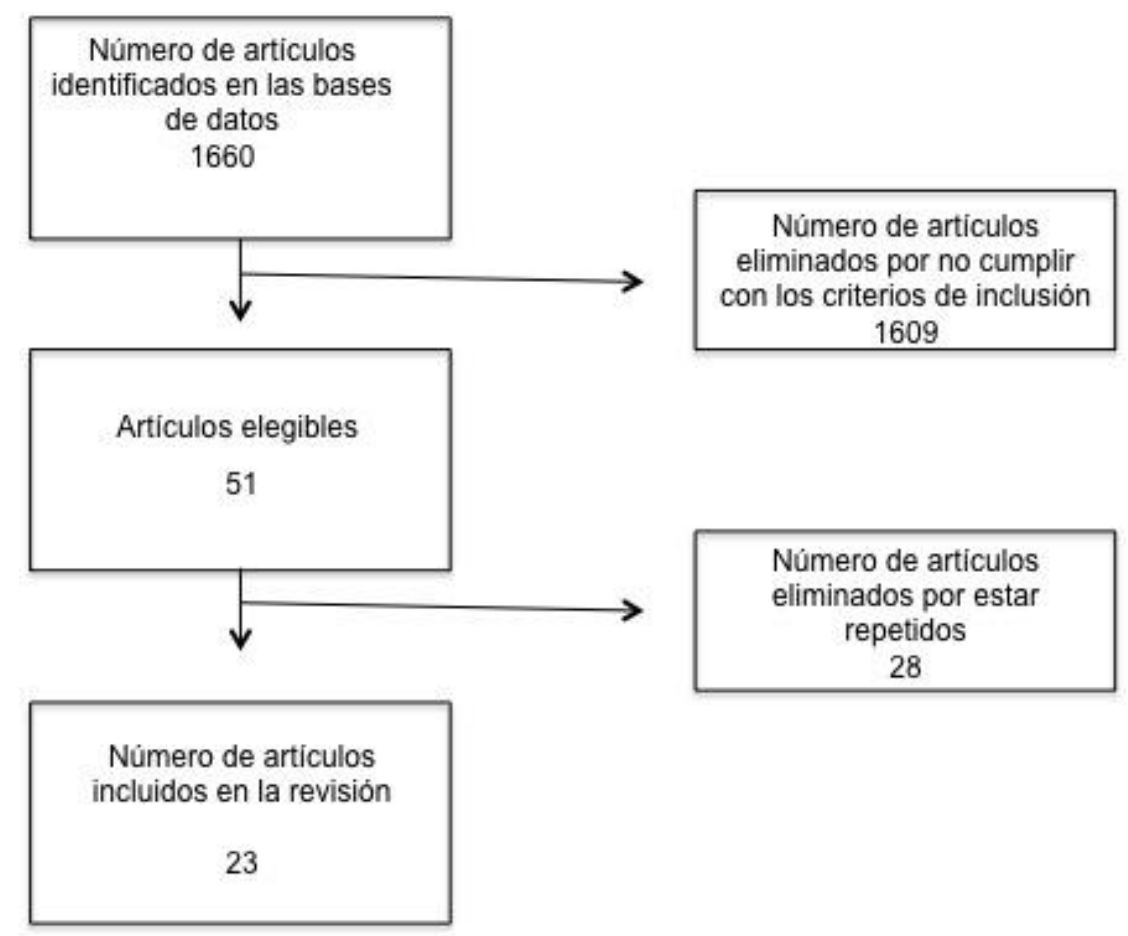

En cuanto a la obsolescencia ${ }^{12}$ de los artículos revisados, de acuerdo con el índice Burton Kleber fue de 5.5 años, mientras que el índice de Price (porcentaje de artículos con edad inferior a 5 años) fue $43 \%$. El total de artículos estaban publicados en inglés.

El masaje y el DMT

El masaje es definido como un conjunto de movimientos sistemáticos manuales o mecánicos practicados sobre los tejidos blandos del cuerpo, regularmente con fines terapéuticos, ayudando en la reactivación sanguínea y linfática, la relajación muscular, el alivio del dolor, la recuperación del equilibrio metabólico y otros beneficios tanto físicos y como metabólicos ${ }^{13}$.

Por su parte la Real Academia Española ${ }^{14}$ lo define como una "operación consistente en presionar, frotar o golpear rítmicamente y con intensidad adecuada determinadas regiones del cuerpo, principalmente la masa muscular, con fines terapéuticos, deportivos, estéticos, etc." 
Existen más de cien técnicas de masaje, sin embargo, el masaje sueco o clásico es uno de las principales e incluye diversas maniobras entre las que se encuentran: Frotamiento (Effleurage), Amasamiento (Petrissage), Percusión (Tapotement), Fricción (Friction) y Vibración (Shaking) ${ }^{15}$.

Los principales efectos fisiológicos del masaje están centrados en el incremento de temperatura de la piel y el músculo, lo que mejora el rendimiento de éste último por el aumento del flujo sanguíneo ${ }^{13}$. Weerapong et al. ${ }^{16}$ (figura 2) presentan un modelo teórico del mecanismo fisiológico y neurológico (además del biomecánico y psicológico) y como pueden ser afectados por el masaje, y por lo tanto disminuir el DMT.

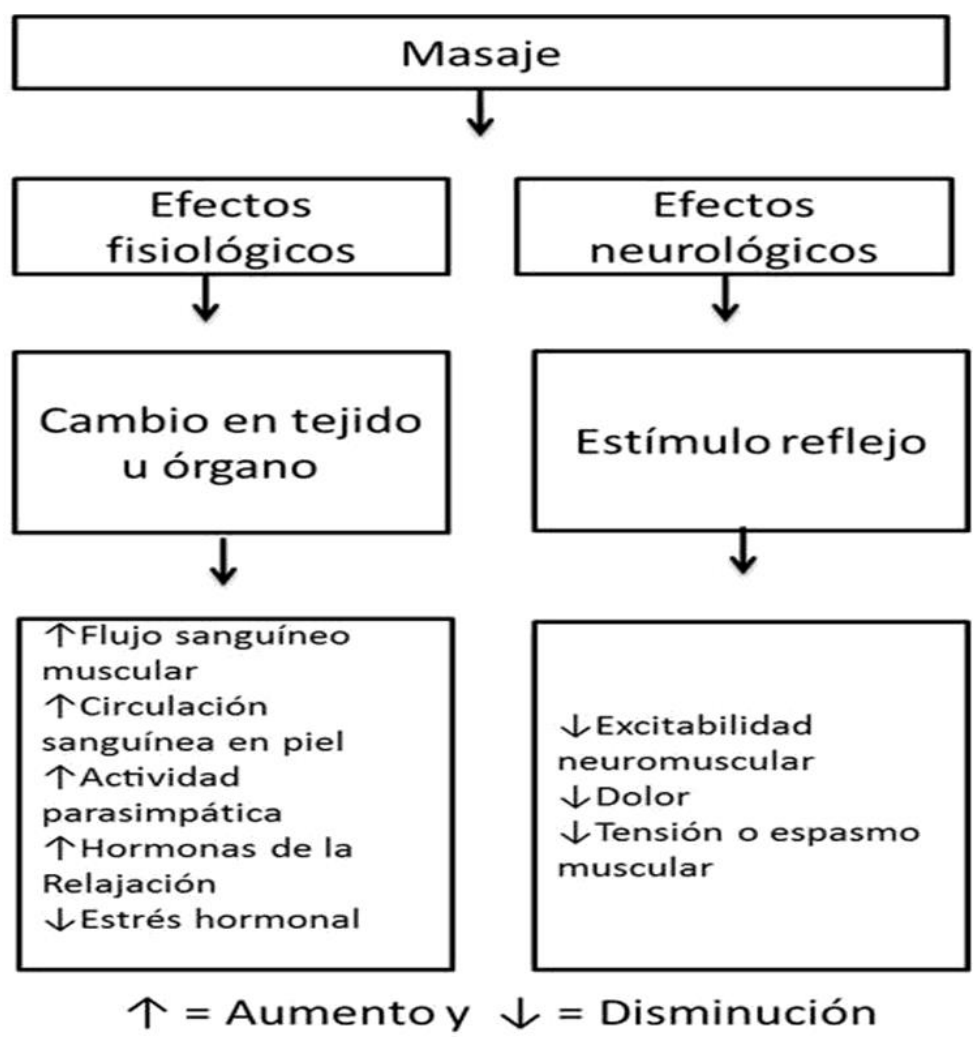

Figura 2. Modelo teórico de los efectos fisiológicos y neurológicos del masaje.

Adaptado de Weerapong et al. (16).

A continuación se presentan los resultados obtenidos en la búsqueda, en primer lugar aquellos estudios donde el masaje tuvo efecto positivo, posteriormente aquellos en los que no hubo efecto o éste fue negativo. Por último 
se presentan las diferentes revisiones del masaje y DMT que se han llevado a cabo en el periodo analizado.

Estudios donde han encontrado efectos positivos del masaje sobre el DMT

Farr et al. ${ }^{17}$ llevaron a cabo un estudio donde aplicaron masaje a una de las piernas sometidas a ejercicio, la otra sirvió de control. Les midieron el dolor muscular en los siguientes cinco días. Entre sus resultados encontraron que el dolor muscular de la pierna tratada disminuyó significativamente a las $24 \mathrm{~h}$ de haber recibido el masaje. Hilbert et al. ${ }^{18}$ investigaron los efectos psicológicos y fisiológicos del masaje sobre el DMT, encontraron que 48 h después de realizar el ejercicio, los sujetos tratados con el masaje presentaban menor intensidad del dolor en comparación con los sujetos del grupo control. Zainuiddin et al. ${ }^{19}$ también usaron el diseño de comparar un miembro tratado y el contralateral sirviendo de control. Sometieron a un grupo de 10 sujetos a una sesión de ejercicio excéntrico para producirles el DMT, tres horas después a uno de los brazos se le aplicó un masaje de diez minutos de duración, encontraron que el dolor muscular en el miembro tratado era menor un 30\% en comparación con el control. Mancinelli et al. ${ }^{20}$ evaluaron el efecto de masaje sobre el DMT en los muslos en mujeres jugadoras de baloncesto. Reportan que la aplicación del masaje aliviaba el dolor muscular y mejoraba el salto vertical en las participantes que recibieron el masaje en comparación con el grupo control. En el estudio de Law et al. ${ }^{21}$ participaron 44 sujetos que fueron sometidos a un ejercicio fatigante, se formaron tres grupos, después del ejercicio a uno se le aplicó masaje superficial a otro masaje profundo, el tercer grupo sirvió de control. Ambos tipos de masaje disminuyeron el dolor muscular comparado con el grupo control. Willems et al. ${ }^{22}$ encontraron reducción del DMT de los cuádriceps con la aplicación del masaje después de una caminata cuesta bajo en tapiz rodante, también vieron que dicha terapia aceleraba la recuperación funcional de los sujetos tratados. Khamwong et al. ${ }^{23}$ determinaron los efectos profilácticos del masaje deportivo sobre los síntomas del daño muscular producido por el ejercicio 
excéntrico de los extensores de la muñeca. El grupo que recibió masaje mostró una tendencia de menor intensidad del dolor muscular en comparación con el grupo control. El mismo tipo de masaje fue utilizado por Boguszewski et al. ${ }^{24}$ en su estudio donde concluyeron que los sujetos tratados se recuperaban más rápido y mejoraban la eficiencia muscular, además de servir como una forma efectiva para el tratamiento del dolor muscular. Han et al. ${ }^{25}$ reportan que el grupo que fue tratado con 15 minutos de masaje en la pantorrilla disminuía significativamente el DMT después de una actividad física agotadora.

Andersen et al. ${ }^{26}$ compararon los efectos del masaje y el ejercicio activo en el alivio del dolor muscular. Los resultados fueron que después de la intervención, en ambos casos hubo disminución del dolor muscular de los hombros tratados en comparación con los no tratados. Pilladi et al. ${ }^{27}$ también compararon el masaje con el ejercicio activo y encontraron que el masaje en el cuádriceps era más efectivo para disminuir el DMT que el ejercicio activo. Por su parte Imtiyaz et al. ${ }^{28}$ compararon los efectos de la terapia de vibraciones y el masaje en la prevención del DMT, para lo cual formaron tres grupos, a uno de ellos se aplicó vibraciones, a otro masaje y el tercero sirvió de control. El dolor muscular se presentó en los tres grupos, sin embargo, en los dos grupos experimentales fue significativamente menor que el grupo control. El masaje con hielo ha sido comparado con la inmersión en agua helada en deportistas de élite y se ha concluido que el primero es más efectivo en la relajación del músculo y en la sensación del DMT ${ }^{29}$. Recientemente Kargarfard et al. ${ }^{30}$ Llevaron a cabo un estudio con un grupo de fisicoculturistas aplicándoles masaje después de producir el DMT, encontraron que el grupo que recibió el tratamiento el dolor físico se redujo a las 24 horas.

El sistema LPG® (iniciales de su creador, Louis Paul Guitay) es una técnica de masaje donde se utiliza un aparato que usa el efecto del vacío generado por presión negativa para actuar sobre la pie| ${ }^{31}$. Este sistema mecánico permite que la terapia de masaje sea aplicada más rápida y consistentemente ${ }^{32}$. Kianmarz ${ }^{33}$ examinó el sistema de masaje LPG, su objetivo fue determinar sus 
efectos sobre el DMT y el desempeño muscular después del ejercicio. Los sujetos que recibieron el masaje disminuyeron significativamente el dolor muscular, la actividad de la creatincinasa, y la circunferencia de las piernas, también recuperaron la fuerza, el salto vertical y la flexibilidad más rápido en comparación con el grupo control.

Otro dispositivo usado para aplicar el masaje son los rodillos de polietileno o polivinilo los cuales permiten a la persona aplicarse automasajes. De acuerdo con Sefton ${ }^{34}$, la fricción creada durante el balanceo con el rollo causa un incremento de la temperatura de la parte tratada y posiblemente cambia a un estado más fluido. Jay et al. ${ }^{35}$ usaron un rodillo de espuma de polietileno para aplicar el masaje a los sujeto de su estudio y encontraron que el dolor muscular era menor que el de los sujetos del grupo control. MacDonald et al. ${ }^{36}$ también usaron un rodillo de espuma para aplicar el masaje a los sujetos de su estudio. Vieron que el dolor disminuía considerablemente y mejoraba el rango de movimiento de la cadera de los sujetos tratados con dicho instrumento. Recientemente Pearcey et al. ${ }^{37}$ encontraron que con el uso del rodillo de polivinilo se mejoraba la recuperación, también reportan que el decremento del desempeño físico era menor después de la producción del DMT. 


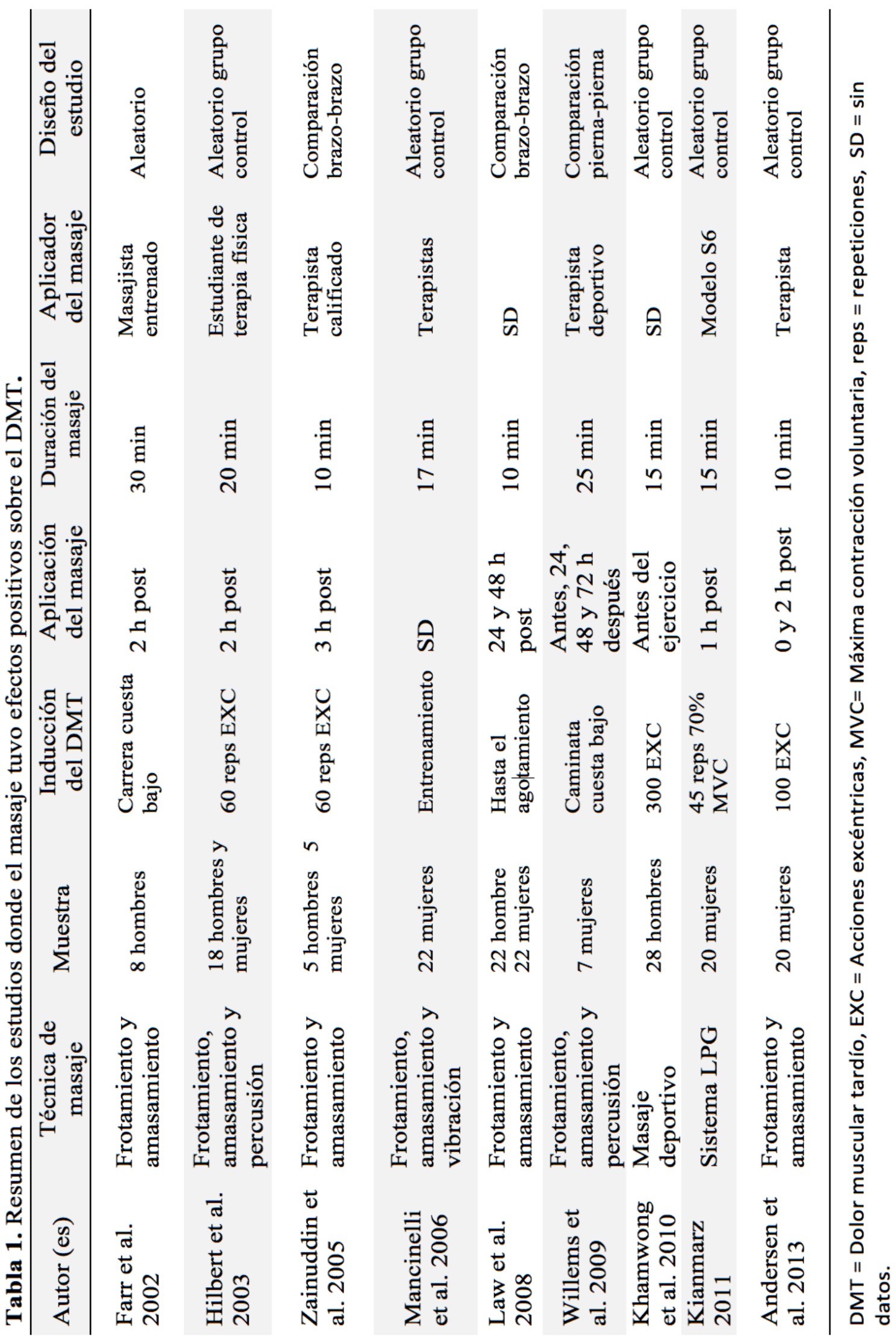




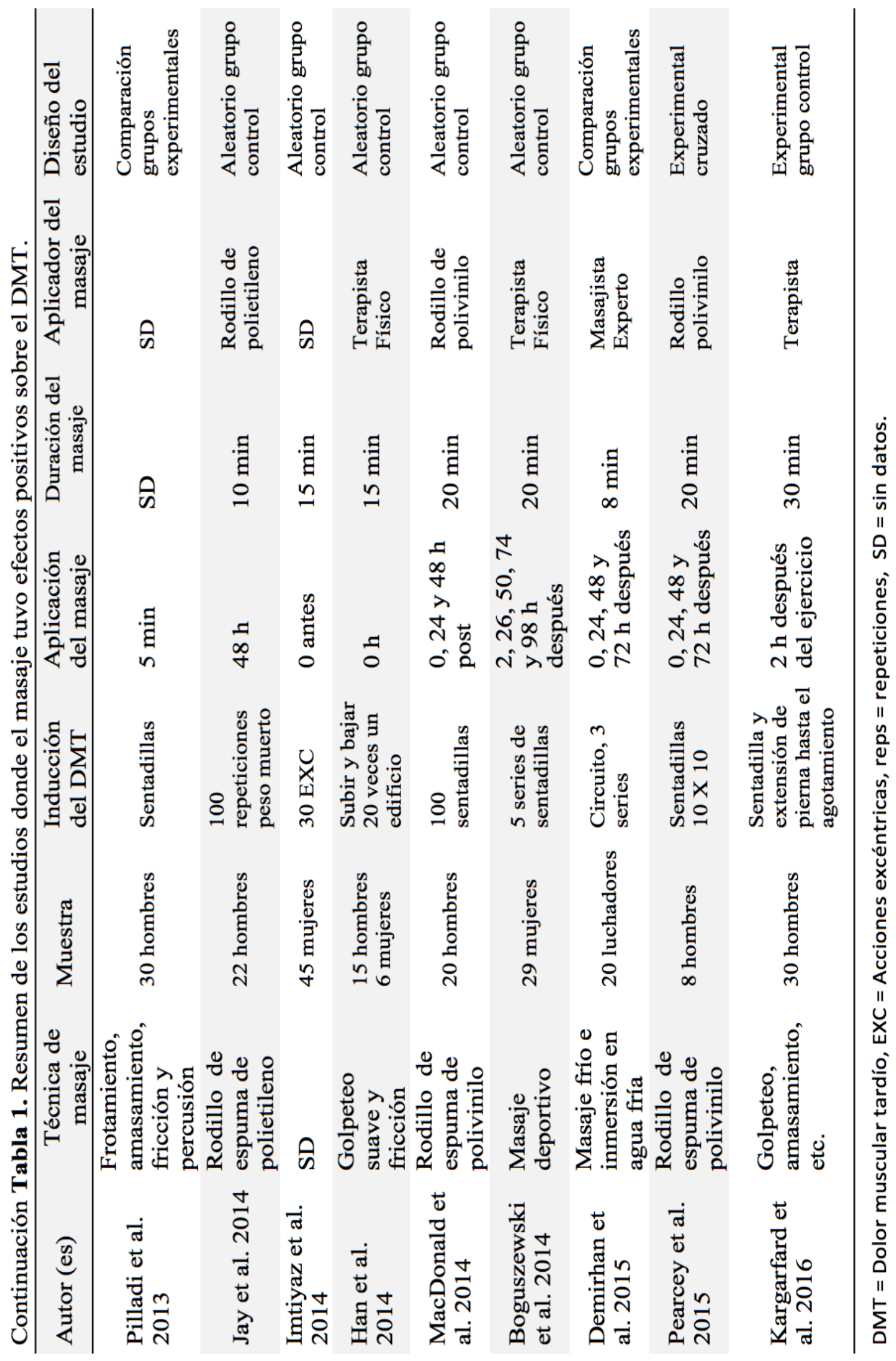


Estudios donde no se han encontrado efectos positivos del masaje en el $D M T$

Jonhagen et al. ${ }^{38}$ realizaron un estudio cuyo objetivo fue examinar la efectividad del masaje deportivo en la reducción del DMT y otras variables. Los sujetos que participaron eran atletas recreacionales. Se les aplicó masaje deportivo en una pierna (la otra sirvió de control), no encontraron diferencia en la sensación de dolor entre la pierna tratada y la control de los sujetos participantes. Dawson et al. ${ }^{39}$ evaluaron los efectos de la terapia de masaje sobre la fuerza, hinchazón y dolor muscular después de un medio maratón, participaron en el estudio 12 corredores recreacionales, a quienes se les aplicó masaje en una de las piernas (la otra sirvió de control) uno, cuatro, ocho y once días después de la carrera. Encontraron que el masaje no afectaba en la recuperación de las variables medidas.

El objetivo del estudio conducido por Hart et al. ${ }^{40}$ fue determinar los efectos de un tratamiento de masaje deportivo de corta duración sobre la hinchazón y el dolor muscular. No encontraron interacción entre el tratamiento y el tiempo para la circunferencia y el dolor del miembro evaluado y no se vio ningún efecto del tratamiento. Por su parte Abad et al. ${ }^{41}$ hicieron un estudio donde formaron tres grupos, el primero recibió masaje y no hizo ejercicio, el segundo realizó ejercicio y masaje, el tercero únicamente hizo el ejercicio. Encontraron incremento del dolor, reducción del rango de movimiento articular (ROM) y de la fuerza después del ejercicio pero no encontraron diferencia entre el segundo y tercer grupo. En un estudio piloto Micklewright ${ }^{42}$ evaluó la técnica de masaje liberación de tejido blando y sus efectos sobre el DMT, la circunferencia y el ROM del miembro tratado. Concluyó que esta técnica agravaba el DMT. Por lo que para deportistas o rehabilitadores que buscan un alivio rápido del DMT es importante utilizar una técnica menos agresiva. 
Tabla 2. Resumen de los estudios donde no encontraron efectos positivos del masaje sobre el DMT.

\begin{tabular}{|c|c|c|c|c|c|c|c|}
\hline Autor (es) & $\begin{array}{c}\text { Técnica de } \\
\text { masaje }\end{array}$ & Muestra & $\begin{array}{l}\text { Inducción del } \\
\text { DMT }\end{array}$ & $\begin{array}{l}\text { Aplicación } \\
\text { del masaje }\end{array}$ & $\begin{array}{c}\text { Duración del } \\
\text { masaje }\end{array}$ & $\begin{array}{c}\text { Aplicador del } \\
\text { masaje }\end{array}$ & $\begin{array}{c}\text { Diseño del } \\
\text { estudio }\end{array}$ \\
\hline $\begin{array}{l}\text { Jonhagen et } \\
\text { al. } 2004\end{array}$ & Deportivo & $\begin{array}{l}8 \text { hombres } 8 \\
\text { mujeres }\end{array}$ & $300 \mathrm{EXC}$ & $\begin{array}{l}10 \text { min, } 1 \text { y } \\
2 \text { días post }\end{array}$ & $10 \mathrm{~min}$ & $\begin{array}{l}\text { Terapista } \\
\text { fisico }\end{array}$ & $\begin{array}{l}\text { Comparación } \\
\text { pierna-pierna }\end{array}$ \\
\hline $\begin{array}{l}\text { Dawson et al. } \\
2004\end{array}$ & $\begin{array}{l}\text { Frotamiento y } \\
\text { amasamiento }\end{array}$ & $\begin{array}{l}8 \text { hombres } \\
4 \text { mujeres }\end{array}$ & $\begin{array}{l}\text { Medio } \\
\text { maratón }\end{array}$ & $\begin{array}{l}1,4,8 \text { y } 11 \\
\text { días post }\end{array}$ & $30 \mathrm{~min}$ & Terapista & $\begin{array}{l}\text { Comparación } \\
\text { pierna-pierna }\end{array}$ \\
\hline $\begin{array}{l}\text { Hart et al. } \\
2005\end{array}$ & Deportivo & $\begin{array}{l}10 \text { hombres } 9 \\
\text { mujeres }\end{array}$ & $\begin{array}{l}4-5 \text { series de } \\
35 \text { reps EXC }\end{array}$ & $\begin{array}{l}24,48 \text { y } 72 \mathrm{~h} \\
\text { post }\end{array}$ & $5 \mathrm{~min}$ & $\begin{array}{l}\text { Entrenador } \\
\text { certificado }\end{array}$ & $\begin{array}{l}\text { Comparación } \\
\text { pierna-pierna }\end{array}$ \\
\hline $\begin{array}{l}\text { Micklewright } \\
2009\end{array}$ & $\begin{array}{l}\text { Liberación del } \\
\text { tejido blando }\end{array}$ & 20 hombres & $80 \mathrm{EXC}$ & $\begin{array}{l}\text { Después de } \\
\text { medir el } \\
\text { dolor }\end{array}$ & $\begin{array}{l}30 \\
\text { Compresiones }\end{array}$ & $\begin{array}{l}\text { Terapista } \\
\text { calificado }\end{array}$ & $\begin{array}{l}\text { Aleatorio } \\
\text { grupo control }\end{array}$ \\
\hline $\begin{array}{l}\text { Abad et al. } \\
2010\end{array}$ & Clásico & 18 hombres & $30 \mathrm{EXC}$ & $\mathrm{Oh}$ & $6 \mathrm{~min}$ & Terapista & Grupo control \\
\hline
\end{tabular}

DMT $=$ Dolor muscular tardío, EXC $=$ Acciones excéntricas, reps $=$ repeticiones, $S D=$ sin datos.

\section{Otras revisiones}

Aparte de incluir estudios originales, en este trabajo se incluyen las revisiones que se han llevado a cabo en el periodo analizado, esto con el fin de tener una visión más amplia de las investigaciones de los efectos del masaje en la prevención y tratamiento del DMT. Las revisiones son clasificadas en este trabajo en narrativas y sistemáticas, aunque algunas están presentadas de forma mixta.

En su revisión Hemmings et al..$^{43}$ concluyeron que existen dudas acerca de la efectividad del masaje en DMT ya que hay pocos estudios. Ese mismo año (2001) Chiu et al. ${ }^{44}$ señalan que los estudios del efecto del masaje post-ejercicio parecen rechazar que el masaje manual es benéfico en la reducción del DMT. Connolly et al.6 sugieren que existe poca evidencia científica para apoyar la efectividad del masaje en el tratamiento del DMT. Hilbert et al. ${ }^{45}$ encontraron que en el $75 \%$ de artículos revisados el masaje clásico disminuyó el dolor muscular. Hume et al. ${ }^{7}$ llevaron a cabo un estudio donde examinaron la efectividad de diferentes estrategias para el tratamiento del DMT, entre ellas el masaje. Concluyeron que el masaje presenta resultados variados debido a al tiempo de aplicación y a la técnica utilizada. En su revisión Weerapong et al. ${ }^{16}$ analizaron 
nueve artículos relacionados con el masaje y el DMT, mencionan que los datos no son concluyentes debido a que la mayoría de los estudios usan muestras pequeñas, algunos estudios usan el miembro contralateral como control, lo que pudiera introducir un sesgo intrasujeto, utilización de diferentes técnicas y otras limitaciones adicionales. Por su parte Moraska ${ }^{46}$ analizó el masaje deportivo y su efecto sobre diversas variables, entre ellas el DMT. Entre los resultados encontrados fue que el dolor muscular es disminuido con el masaje, aunque no está claro si ayuda a la recuperación de la fuerza más rápidamente. Best et al. ${ }^{47}$ también determinaron la efectividad del masaje deportivo para mejorar la recuperación después de un ejercicio extenuante. Concluyen que los estudios aleatorizados demuestran mayor evidencia de la efectividad del masaje en la recuperación y la disminución del DMT que los estudios de caso. Howatson y Van Someren ${ }^{48}$ llevaron a cabo una revisión del uso de diversas estrategias para la prevención y tratamiento del daño muscular producido por el ejercicio. Reportan que la mayoría de la evidencia apunta hacia los beneficios del masaje en la disminución del dolor muscular, aunque su efecto sobre la función y el desempeño no son claros. Torres et al..$^{49}$ evaluaron si las modalidades usadas en fisioterapia como masaje, crioterapia, estiramiento y ejercicio de baja intensidad eran efectivas para el tratamiento de los síntomas del daño muscular producido por el ejercicio. Encontraron que el masaje era la única intervención con efectos positivos en la reducción del dolor muscular. Recientemente Nelson ${ }^{50}$ realizó un estudio donde revisó el uso del masaje como terapia para el tratamiento del DMT. Concluye que el masaje ha sido estudiado como una opción en el tratamiento del DMT con resultados prometedores en la reducción del dolor muscular.

\section{CONCLUSIONES}

Después del análisis de los diferentes estudios incluidos en la presente revisión, se puede concluir que en los últimos años el masaje ha mostrado ser una terapia efectiva para disminuir el DMT. Sin embargo, dicha conclusión habría que tomarla con precaución ya que en los estudios existe gran variedad en la 
duración de la aplicación del masaje, de técnicas usadas y sobre todo el diseño de los estudios y el tamaño de las muestras utilizadas. Por último y no menos importante, que el masaje es aplicado por personas y aun cuando sean profesionales en la terapia, puede existir una gran variabilidad en la ejecución.

En cuanto a las revisiones se concide con la mayoría de ellas en el sentido de que se requieren más estudios para poder determinar de una forma más precisa el efecto del masaje sobre el DMT.

\section{Consideraciones finales}

Si bien a lo largo de estos últimos quince años han sido varias las revisiones que se han llevado a cabo sobre el tema aquí tratado, en el presente estudio se ha realizado una actualización, en el que se incluyen todos los estudios que estén acordes con la definición del masaje dada por la Real Academia Española por lo que también se analizaron estudios en los que se usaron implementos para aplicar el masaje. También se incluyó en el análisis dichas revisiones.

\section{Conflictos de interés: ninguno}

\section{REFERENCIAS}

1. Safran MR, Seaber AV, Garrett WE, Jr. Warm-up and muscular injury prevention. An update. Sports Med. 1989; 8:239-49.

2. Nosaka K Muscle soreness and damage and repeated-bout effect. In: Tiidus P, editor. Skeletal muscle damage and repair: mechanisms and interventions. Champaign, IL: Human Kinetics. 2008;p. 59-76.

3. Bubbico A, Kravitz L. Eccentric exercise: A comprehensive review of a distinctive training method. IDEA Fitness Journal. 2010;7:50-9.

4. Heymanson N, Hiskins B. Delayed onset muscle soreness and soft tissue therapywhat makes good research. SportEX Dynamics. 2006;10:8-10.

5. Cheung K, Hume P, Maxwell L. Delayed onset muscle soreness: treatment strategies and performance factors. Sports Med. 2003;33:145-64.

6. Connolly DA, Sayers SP, McHugh MP. Treatment and prevention of delayed onset muscle soreness. J Strength Cond Res. 2003;17:197-208. 
7. Hume PA, Cheung K, Maxwell L, Weerapong P. DOMS: An overview of treatment strategies. International SportMed Journal. 2004;5:98-118.

8. Candia-Luján R, de Paz-Fernández JA. ¿Son efectivos los antiinflamatorios no esteroides en el tratammmiento del dolor muscular tardìo? CienciaUAT. 2014;9:7683.

9. Luden ND, Saunders MJ, Todd MK. Postexercise carbohydrate-protein- antioxidant ingestion decreases plasma creatine kinase and muscle soreness. Int J Sport Nutr Exerc Metab. 2007; 17:109-23.

10. Vaile J, Halson S, Gill N, Dawson B. Effect of hydrotherapy on the signs and symptoms of delayed onset muscle soreness. Eur J Appl Physiol. 2008;102:447-55.

11. Guirao-Goris JA, Olmedo Salas, Ferrer Ferrandis E. El artículo de revisión. Revista Iberoamerícana de Enfermería Comunitaria. 2008;1:1-25.

12. Vásquez-Morales A, Wanden-Berghe C, Sanz-Valero J. Ejercicio físico y suplementos nutricionales, efecto de un uso combinado en las personas mayores de 65 años; una revisión sistemática. NutrHosp. 2014;28:107-14

13. Beck MF. Masaje Terapèutico. Teorìa y Pràctica. Madrid: Paraninfo 2000.

14. Real Academia Española. Diccionario de la Lengua Española. Barcelona: Espasa; 2014.

15. Lewis $M$, Johnson MI. The clinical effectiveness of therapeutic massage for musculoskeletal pain: a systematic review. Physiotherapy. 2006;92:146-58.

16. Weerapong $P$, Hume PA, Kolt GS. The mechanisms of massage and effects on performance, muscle recovery and injury. Sports Med. 2005;35:235-56.

17. Farr T, Nottle C, Nosaka K, Sacco P. The effects of therapeutic massage on delayed onset muscle soreness and muscle function following downhill walking. J Sci Med Sport. 2002;5:297-306.

18. Hilbert JE, Sforzo GA, Swensen T. The effects of massage on delayed onset muscle soreness. Br J Sports Med. 2003;37:72-5.

19. Zainuddin Z, Newton M, Sacco P, Nosaka K. Effects of massage on delayed-onset muscle soreness, swelling, and recovery of muscle function. J Athl Train. 2005;40(3):174-80.

20. Mancinelli CA, Davis DS, Aboulhosn L, Brady M, Eisenhofer J, Foutty S. The effects of massage on delayed onset muscle soreness and physical performance in female collegiate athletes. Phys Ther Sport. 2006;7:5-13. 
21. Law LAF, Evans S, Knudtson J, Nus S, Scholl K, Sluka KA. Massage reduces pain perception and hyperalgesia in experimental muscle pain: a randomized, controlled trial. J Pain. 2008 9:714-21.

22. Willems MET, Hale T, Wilkinson CS. Effects of manual massage on muscle-specific soreness and single jump performance after downhill treadmill walking. Medicina Sportiva. 2009; 13:61-6.

23. Khamwong P, Pirunsan U, Paungmali A. The prophylactic effect of massage on symptoms of muscle damage induced by eccentric exercise of the wrist extensors. Journal of Sports Sciences and Technology. 2010;10:245-59.

24. Boguszewski D, Szkoda S, Adamczyk JG, Bialoszewski D. Sport massage therapy on the reduction of delayed onset muscle soreness of the quadriceps femoris. Human Movement. 2014;15: 234-37.

25. Han JH, Kim MJ, Yang HJ, Lee YJ, Sung YH (2014) Effects of therapeutic massage on gait and pain after delayed onset muscle soreness. J Exerc Rehabil. 2014;10:13640.

26. Andersen LL, Jay K, Andersen CH, Jakobsen MD, Sundstrup E, Topp R, et al. Acute effects of massage or active exercise in relieving muscle soreness: randomized controlled trial. J Strength Cond Res. 2013;27:3352-59.

27. Pilladi AC, Apparao P, Chakravarthi CS, Sekshar PKC. Effects of massage vs active exercises on experimentally induced delayed onset of muscle soreness. International Journal of Physiotherapy and Research. 2013;1:238-42.

28. Imtiyaz S, Veqar Z, Shareef MY. To compare the effect of vibration therapy and massage in prevention of delayed onset muscle soreness (DOMS). J Clin Diagn Res. 2014;8:133-6.

29. Demirhan B, Yaman M, Cengiz A, Saritas N, Günay M. Comparison of ice massage versus cold-water immersion on muscle damage and DOMS levels of elite wrestlers. Anthropologist. 2015;19:123-9.

30. Kargarfard M, Lam ET, Shariat A, Shaw I, Shaw, BS, Tamrin, SB. Efficacy of massage on muscle soreness, perceived recovery, physiological restoration and physical performance in male bodybuilders. Journal of Sports Sciences. 2016; 34:959-65.

31. Bourgeois JF, Gourgou S, Kramar A, Lagarde JM, Guillot B. A randomized, prospective study using the $L P G \circledast$ technique in treating radiation-induced skin fibrosis: Clinical and profilometric analysis. Skin Res Technol. 2008;14:71-6.

32. Gulec AT. Treatment of cellulite with LPG endermologie. Int J Dermatol. 2009;48:265- 70 . 
33. Kianmarz V. The effects of LPG massage system on delayed onset muscle soreness and muscular performance after resistance exercise. HealthMED. 2011;5:1474-1478.

34. Sefton J. Myofascial release for the athletic trainers, Part I: Theory and session guidelines. Athl Ter Today. 2004;9:48-9.

35. Jay K, Sundstrup E, Sondergaard SD, Behm D, Brandt M, Saervoll CA, et al. Specific and cross over effects of massage for muscle soreness: randomized controlled trial. Int J Sports Phys Ther. 2014; 9:82-91.

36. Macdonald GZ, Button DC, Drinkwater EJ, Behm DG. Foam rolling as a recovery tool after an intense bout of physical activity. Med Sci Sports Exerc. 2014;46:131-42.

37. Pearcey GEP, Bradbury-Squires DJ, Kawamoto JE, Drinkwater EJ, Behm DG, Button DC. Foam rolling for delayed-onset muscle soreness and recovery of dinamic performance measures. J Athl Train. 2015; 50: 5-13.

38. Jönhagen S, Ackermann P, Eriksson T, Saartok T, Renström PA. Sports massage after eccentric exercise. Am J Sports Med. 2004; 32:1499-503.

39. Dawson LG, Dawson KA, Tiidus PM. Evaluating the influence of massage on leg strength, swelling, and pain following a half-marathon. J Sports Sci Med. 2004; 3:3743.

40. Hart JM, Swanik CB, Tierney RT. Effects of sport massage on limb girth and discomfort associated with eccentric exercise. J Athl Train. 2005; 40:181-5.

41. Abad CCC, Ito LT, Barroso R, Ugrinowitsch C, Tricoli V. Effect of classical massage on subjective perceived soreness, edema, range of motion and maximum strength after delayed onset muscle soreness induced by exercise. Rev Bras Med Esporte. 2010;16:36-40.

42. Micklewright $D$. The effect of soft tissue release on delayed onset muscle soreness: a pilot study. Phys Ther Sport. 2009;10:19-24.

43. Hemmings BJ. Physiological, psychological and performance effects of massage therapy in sport: A review of the literature. Phys Ther Sport. 2001; 2:165-70.

44. Chiu LZ, Weiss LW, Fry AC. Post-training massage: a review for strength and powerathletes. / Massage post entrainement: apercu pour les athletes de force et puissance. Strength Cond J. 2001;23:65-9.

45. Hilbert JE, Sforzo GA, Swensen T. The role of massage in the treatment of delayed onset muscle soreness: A brief review. International SportMed Journal. 2004;5:1109.

46. Moraska A. Sports massage. A comprehensive review. J Sports Med Phys Fitness. 2005;45:370-80. 
47. Best TM, Hunter R, Wilcox A, Haq F. Effectiveness of sports massage for recovery of skeletal muscle from strenuous exercise. Clin J of Sport Med. 2008;18:446-60.

48. Howatson G, van Someren KA. The prevention and treatment of exercise induced muscle damage. Sports Med. 2008;38:483-503.

49. Torres R, Ribeiro F, Alberto Duarte J, Cabri JM. Evidence of the physiotherapeutic interventions used currently after exercise-induced muscle damage: systematic review and meta-analysis. Phys Ther Sport. 2012;13:101-14.

50. Nelson N. Delayed onset muscle soreness: Is massage effective? J Bodyw Mov Ther. 2013;17:475-82.

Referencias totales citadas: 50

Referencia citadas correspondientes a la Rev Ib CC Act Dep: 0 